\title{
Optimal Screening in Structured SIR Epidemics
}

\author{
B. Ainseba ${ }^{1}$, M. Iannelli2* \\ ${ }^{1}$ Bordeaux University, Institut de Mathématiques de Bordeaux, UMR CNRS 5251, Case 26 \\ Université Bordeaux Ségalen, 3 Place de la Victoire, 33076 Bordeaux Cedex, France \\ 2 Department of mathematics, University of Trento, 38050 Povo, Trento, Italy
}

\begin{abstract}
We present a model for describing the spread of an infectious disease with public screening measures to control the spread. We want to address the problem of determining an optimal screening strategy for a disease characterized by appreciable duration of the infectiveness period and by variability of the transmission risk. The specific disease we have in mind is the HIV infection. However the model will apply to a disease for which class-age structure is significant and should not be disregarded.
\end{abstract}

Keywords and phrases: PDE in connection with biology, population dynamics, epidemiology, optimal control problems involving partial differential equations

Mathematics Subject Classification: 35Q92, 92D25, 92D30, 49J20

\section{Introduction}

As proved in the last decades, the transmission dynamics of infectious diseases is an area of applied sciences in which the use of mathematical models has allowed great advances, not only on the theoretical side, but also on the side of the management of actual public health policies. Within this context, recent interest has been devoted to the problem of epidemics control and some mathematical modeling has been consequently developed in order to determine suitable strategies for disease defeat or at least for keeping as low as possible the costs of an epidemic outbreak.

From a sanitary point of view different actions are possible in order to control a given disease, but each disease has its own specific features that require specific type of intervention. Thus vaccination, screening, tracing, quarantine, are different possible measures that should be optimized according with the specific disease under study.

A direct way to test control strategies is to produce simulation of a model, varying parameters within a class of planned interventions, comparing different outcomes and discussing convenient choices (see for instance [1], [5], [14], [20], [23], [24], [26], [27], [30], for recent samples of this approach). On the other hand, a less empirical approach consists in resorting to mathematical control theory in order to find optimal strategies, minimizing some cost function related to sanitary objectives and social limits.

Concerning this research trend, early optimal control results can be found in [25], [29], [31], [32], [33], and refer to the basic SIS and SIR models by Kermack and McKendrick. In these papers the ODE theory is applied in order to identify optimal removal strategies for susceptibles or infectives, corresponding to

\footnotetext{
${ }^{*}$ Corresponding author. E-mail: bedreddine.ainseba@u-bordeaux2.fr
} 
immunization programs or quarantine and screening. In a more recent paper [8] these results are extended to a more general epidemic model and several control measures are included in the scheme. Other recent work has been also based on this approach for modeling various aspects of epidemic control [4], [9], [15], $[21],[28]$.

All the previous approaches to control strategies are concerned with epidemic models based on the assumption of homogeneous mixing of a homogeneous population, thus disregarding the possible selective effect of population structure. However, control problems within the context of age structured populations have also been considered (see for instance [2], [3], [7], [13], [19]) and epidemic models, structured by variables like chronological age and class age, have been discussed. Some work in this context has been developed in [6], [10], [11], [12], [14] and [16]-[18], [27]; in particular, in [6], principles and methods pertaining to optimal control theory for partial differential equations have been applied.

This paper is devoted to analyse a framework corresponding to diseases for which class-age structure is significant and should not be disregarded. Namely we want to address the problem of determining a screening strategy for a disease characterized by appreciable duration of the infectiveness period and by variability of the transmission risk. The specific disease we have in mind is HIV/AIDS (see for instance a recent contribution [20]). However the model will apply to any disease (not only human diseases) with the following specific features

- individuals who catch the disease stay active and infective, with variable infectiveness, untill they are identified as carriers, or die or become inactive due to the disease;

- infective individuals may be identified as carriers because of the natural progression of the disease that produces symptoms or because they participate in a screening program;

- once they are identified as carriers, they are partly removed in the sense that a fraction of them stops to be infective, either by recovering and becoming immune or by treatment and prevention measures; the remaining part of the screened infectives continues to belong to the class of infectives because it does not really recover or disregards prevention measures.

- the processes described above depend on the time elapsed since infection so that infectiveness depends on the pathogenic agent progression according to the disease features, the success of the treatment is higher for earlier stages of the disease, while the death rate is higher for later ones.

Next section is devoted to the setting of the model on the basis of the features described above, while in Section 3 we analyze the dynamics of the model determining steady states and their stability. In Section 4 we discuss the cost function and in Section 5 we prove existence of an optimal screening strategy. Finally, in Section 6 we derive the optimality conditions and use them to perform some simulations to compute the optimal strategy in connection with different values of the parameters.

\section{Setting of the model}

Let $S(t), i(\theta, t), R(t)$ describe the status of the population in the usual epidemic classes of susceptibles, infected, removed individuals. Namely $S(t)$ and $R(t)$ denote the number of individuals in the corresponding class, while $i(\theta, t)$ denotes the density of individuals with respect to $\theta$ which is the age of infection, i.e. the time elapsed since the individual was infected. We assume a simple demographic process defined by constant rates, namely by the two parameters

$-\Lambda=$ demografic recruitment rate of susceptibles;

$-\mu=$ demographic mortality rate; 
then the classical S-I-R model structured by the age of infection and including a screening strategy is governed by the following system

$$
\left\{\begin{array}{l}
\text { a) } S^{\prime}(t)=\Lambda-\lambda(t) S(t)-\mu S(t) \\
\text { b) } \frac{\partial i}{\partial t}+\frac{\partial i}{\partial \theta}+[\mu+\nu(\theta)+\gamma(\theta)+v(t) \Psi(N(t)) \phi(\theta)] i(\theta, t)=0 \\
\text { c) } R^{\prime}(t)=\int_{0}^{\theta_{m}}[\gamma(\theta)+v(t) \Psi(N(t)) \phi(\theta)] i(\theta, t) d \theta-\mu R(t) \\
\text { d) } N(t)=S(t)+\int_{0}^{\theta_{m}} i(\theta, t) d \theta+R(t)
\end{array}\right.
$$

endowed with the following incidence condition

$$
i(0, t)=\lambda(t) S(t)
$$

and the initial conditions

$$
S(0)=S_{0}, \quad i(\theta, 0)=i_{0}(\theta), \quad R(0)=R_{0} .
$$

In (2.1) the different parameters have the following meaning

$-\gamma(\theta)=$ age-dependent removal rate due to the natural course of the disease;

$-\nu(\theta)=$ age-dependent extra removal rate accounting for deaths or isolation due to the disease;

$-v(t)=$ screening strategy, i.e. the planned fraction of individuals to be screened per unit of time;

$-\Psi(x)=$ population-dependent individual functional response to the screening offer, i.e. the fraction of individuals accepting the screening offer;

- $\phi(\theta)=$ age-dependent disease detectability and successful treatment.

The three age-dependent functions $\gamma(\theta), \nu(\theta)$ and $\phi(\theta)$ are all non-negative and defined in the finite interval $\left[0, \theta_{m}\right]$, where $\theta_{m}$ denotes the maximal age of the disease before removal. In order to have all infected individuals removed before $\theta_{m}$ we assume

$$
\int_{0}^{\theta_{m}} \gamma(\theta) d \theta=+\infty .
$$

Finally, in $(2.1, a)$ and $(2.2), \lambda(t)$ is the force of infection and is assumed to have the following constitutive form

$$
\lambda(t)=\frac{c}{N(t)} \int_{0}^{\theta_{m}} \omega(\theta) i(\theta, t) d \theta
$$

where

$-c=$ contact rate;

$-\omega(\theta)=$ infectiveness of a contact, depending on the disease advancement.

We note that in $(2.1, d), N(t)$ denotes the total active population. In fact, the class of removed individuals includes not only those who have caught the disease and now are recovered and immune, but also those who, though identified as carriers, are not infective by treatment and prevention. All these individuals can be considered as fully active.

In the specific case of HIV this assumption is not unrealistic because seropositive individuals, though carrying the disease and being infective, are assumed not to infect new people since their awareness suggests prevention measures. In this case, in the definition of the function $\phi(\theta)$, successful treatment means successful application of prevention measures. Of course, those individuals who, though screened, do not follow prevention measures, remain in the class of infective seropositive. 
Infected individuals also contribute to the active population before dying or being isolated, in fact the extra mortality $\nu(\theta)$ accounts for the death rate due to the disease but also for a removal rate due to isolation.

We also note that in system (2.1), the factor $\gamma(\theta) i(\theta, t)$ accounts for the density of infected individuals who are removed per unit of time because they develop symptoms (and are treated) or recover and are immune, while the term

$$
v(t) \Psi(N(t)) \phi(\theta) i(\theta, t) d \theta=v(t) \Psi(N(t)) N(t) \phi(\theta) \frac{i(\theta, t)}{N(t)} d \theta
$$

gives the density of infected individuals, with age $\theta$, who are detected by screening and cured per unit of time, (consequently removed and still active). In fact in (2.5), while the factor $v(t) \geq 0$ is the screening strategy, the factor $\Psi(N(t)) N(t)$ accounts for the part of the population effectively accepting to be screened, $\frac{i(\theta, t) d \theta}{N(t)}$ is the probability that the screened individual is actually infective and $\phi(\theta)$ accounts for the disease evidence and is an increasing function of the age $\theta$. We note that concerning the functional response $\Psi(x) x$ we have in mind a Holling type function, though the analogy with the predator-prey mechanism may not be quite adequate. Thus $\Psi(x)$ should be decreasing, while $\Psi(x) x$ should be a saturating increasing function.

We note that it is convenient to adopt the three variables $N(t), S(t)$ and

$$
u(\theta, t)=\frac{i(\theta, t)}{\Gamma(\theta)}, \quad \Gamma(\theta)=e^{-\int_{0}^{\theta} \gamma(s) d s},
$$

that are sufficient to treat problem (2.1). Namely we can focus on the following problem

$$
\left\{\begin{array}{l}
\text { a) } S^{\prime}(t)=\Lambda-\lambda(t) S(t)-\mu S(t) \\
\text { b) } \frac{\partial u}{\partial t}+\frac{\partial u}{\partial \theta}+[\mu+\nu(\theta)+v(t) \Psi(N(t)) \phi(\theta)] u(\theta, t)=0 \\
\text { c) } N^{\prime}(t)=\Lambda-\mu N(t)-\int_{0}^{\theta_{m}} \nu(\theta) \Gamma(\theta) u(\theta, t) d \theta .
\end{array}\right.
$$

endowed with

$$
u(0, t)=\lambda(t) S(t), \quad \lambda(t)=\frac{c}{N(t)} \int_{0}^{\theta_{m}} \omega(\theta) \Gamma(\theta) u(\theta, t) d \theta .
$$

In fact, once this problem is solved, we may get $R(t)$ from equation $(2.1, \mathrm{c})$. Thus, focusing on $(2.7)$, we perform a change of scale defining the following new non-dimensional variables

$$
\begin{gathered}
\tilde{\theta}=\mu \theta, \quad \tilde{t}=\mu t, \quad \tilde{\theta}_{m}=\mu \theta_{m}, \quad \tilde{c}=\frac{c}{\mu}, \quad \tilde{v}(\tilde{t})=\frac{v(t)}{\mu}, \\
\tilde{\gamma}(\tilde{\theta})=\frac{\gamma(\theta)}{\mu}, \quad \tilde{\nu}(\tilde{\theta})=\frac{\nu(\theta)}{\mu}, \quad \tilde{\lambda}(\tilde{t})=\frac{\lambda(t)}{\mu}, \\
\tilde{\Gamma}(\tilde{\theta})=\Gamma(\theta), \quad \tilde{\omega}(\tilde{\theta})=\omega(\theta), \quad \tilde{\phi}(\tilde{\theta})=\phi(\theta), \\
\tilde{S}(\tilde{t})=\frac{\mu}{\Lambda} S(t), \quad \tilde{N}(\tilde{t})=\frac{\mu}{\Lambda} N(t), \quad \tilde{u}(\tilde{\theta}, \tilde{t})=\frac{u(\theta, t)}{\Lambda}, \quad \tilde{\Psi}(x)=\Psi\left(\frac{\Lambda}{\mu} x\right)
\end{gathered}
$$

and we note that

$$
\tilde{\lambda}(\tilde{t})=\frac{1}{\mu} \lambda\left(\frac{\tilde{t}}{\mu}\right)=\frac{\tilde{c}}{\tilde{N}(\tilde{t})} \int_{0}^{\tilde{\theta}_{m}} \tilde{\omega}(s) \tilde{\Gamma}(s) \tilde{u}(s, \tilde{t}) d s
$$




$$
\tilde{\Gamma}(\tilde{\theta})=\Gamma\left(\frac{\tilde{\theta}}{\mu}\right)=e^{-\int_{0}^{\tilde{\theta}} \tilde{\gamma}(s) d s}, \quad \tilde{i}(\tilde{\theta}, \tilde{t})=\tilde{\Gamma}(\tilde{\theta}) \tilde{u}(\tilde{\theta}, \tilde{t}) .
$$

Then, omitting tildes for simplicity, we get the following non-dimensional problem

$$
\left\{\begin{array}{l}
\text { a) } S^{\prime}(t)=1-\lambda(t) S(t)-S(t) \\
\text { b) } \frac{\partial u}{\partial t}+\frac{\partial u}{\partial \theta}+[1+\nu(\theta)+v(t) \Psi(N(t)) \phi(\theta)] u(\theta, t)=0 \\
\text { c) } N^{\prime}(t)=1-N(t)-\int_{0}^{\theta_{m}} \nu(\theta) \Gamma(\theta) u(\theta, t) d \theta,
\end{array}\right.
$$

with the same conditions (2.8). Actually, we note that $(2.9, b)$ and $(2.8)$, by integration along the characteristic lines, yield

$$
u(\theta, t)=\left\{\begin{array}{ll}
u_{0}(\theta-t) & \text { for } \quad \theta>t \\
\lambda(t-\theta) S(t-\theta) & \text { for } \quad \theta \leq t
\end{array}\right\} \times \mathcal{E}(\theta, t ; v, N)
$$

where

$$
\mathcal{E}(\theta, t ; v, N)=e^{-\int_{0}^{\theta \wedge t}[1+\nu(\theta-\sigma)+v(t-\sigma) \Psi(N(t-\sigma)) \phi(\theta-\sigma)] d \sigma},
$$

thus providing an integral formulation of the problem.

\section{Steady states and stability}

If we assume a constant screening strategy, i.e. a strategy $v(t) \equiv v$ which is not time-dependent, then system (2.7) is endowed with steady states that are solutions of the following problem

$$
\left\{\begin{array}{l}
\text { a) } 1-\lambda S-S=0 \\
\text { b) } u^{\prime}(\theta)+[1+\nu(\theta)+v \Psi(N) \phi(\theta)] u(\theta)=0 \\
\text { c) } u(0)=\lambda S \\
\text { d) } 1-N-\int_{0}^{\theta_{m}} \nu(\theta) \Gamma(\theta) u(\theta) d \theta=0 \\
\text { e) } \lambda N=c \int_{0}^{\theta_{m}} \omega(\theta) \Gamma(\theta) u(\theta) d \theta .
\end{array}\right.
$$

In fact, by a steady state we mean a solution of (2.7) which is constant in time so that we are looking for a solution such that the scalar variables $S(t), N(t)$ are constant and the $\theta$-distribution of infectives is a function $u(\theta), \theta \in\left[0, \theta_{m}\right]$. System (3.1) admits the so called disease-free state

$$
S=1, \quad u(\theta) \equiv 0, \quad N=1,
$$

accounting for the case of a population in which the disease is absent. In addition we are interested in endemic states, i. e. solutions with non-trivial $u(\theta)$, accounting for cases in which a core of infectives is always present and steadily distributed with respect to the age of infection. To look for these states, it is convenient to follow the standard procedure of taking the incidence $\sigma=\lambda S$ as a new variable and to solve $(3.1, \mathrm{~b})$ with the initial condition $(3.1, \mathrm{c})$. In fact we have

$$
u(\theta)=\sigma \Pi(\theta, v \Psi(N))
$$

where

$$
\Pi(\theta, \xi)=\exp \left\{-\theta-\int_{0}^{\theta}[\nu(\tau)+\xi \phi(\tau)] d \tau\right\}
$$


Thus, substituting (3.3) into (3.1), we can reduce the system to the following algebraic problem

$$
\left\{\begin{array}{l}
\text { a) } 1-\sigma-S=0 \\
\text { b) } 1-N-\sigma \mathcal{M}(v \Psi(N))=0 \\
\text { c) } N=\operatorname{cSK}(v \Psi(N))
\end{array}\right.
$$

where we have set

$$
\mathcal{M}(\xi)=\int_{0}^{\theta_{m}} \nu(\theta) \Gamma(\theta) \Pi(\theta, \xi) d \theta, \quad \mathcal{K}(\xi)=\int_{0}^{\theta_{m}} \omega(\theta) \Gamma(\theta) \Pi(\theta, \xi) d \theta .
$$

The previous system (3.4) can be easily solved in the special case $\nu(\theta) \equiv 0$. In fact, in this case the system becomes

$$
\left\{\begin{array}{l}
\text { a) } 1-\sigma-S=0 \\
\text { b) } 1-N=0 \\
\text { c) } N=c S \mathcal{K}(v \Psi(N))
\end{array}\right.
$$

and we get

$$
N^{*}=1, \quad S^{*}=\frac{1}{c \mathcal{K}(v \Psi(1))}, \quad \sigma^{*}=\left(1-\frac{1}{c \mathcal{K}(v \Psi(1))}\right) .
$$

Thus an endemic state is possible if and only if

$$
\mathcal{R}_{0}(v)=c \mathcal{K}(v \Psi(1))>1
$$

Concerning the case with $\nu(\theta)$ not identically vanishing, in order to solve (3.4) we may solve $(3.4, \mathrm{~b})$ and $(3.4, \mathrm{c})$ getting $\sigma$ and $S$ as functions of $N$

$$
\sigma=\frac{1-N}{\mathcal{M}(v \Psi(N))}, \quad S=\frac{N}{c \mathcal{K}(v \Psi(N))}
$$

and, substituting in $(3.4, \mathrm{a})$, we get the following equation for $N$,

$$
\left(1-\frac{1}{\mathcal{M}(v \Psi(N))}\right)+N\left(\frac{1}{\mathcal{M}(v \Psi(N))}-\frac{1}{c \mathcal{K}(v \Psi(N))}\right)=0 .
$$

Thus we just need to find solutions of this equation in the interval $(0,1)$ and substitute them in $(3.7)$ to have a non-trivial solution with positive components for system (3.4).

Now, denoting by $\mathcal{H}(N)$ the function on the left hand side of $(3.8)$, since $\mathcal{M}(\xi)<1$ for any $\xi>0$, we have that

$$
\mathcal{H}(0)=\left(1-\frac{1}{\mathcal{M}(v \Psi(0))}\right)<0
$$

On the other hand

$$
\begin{aligned}
\mathcal{H}^{\prime}(N)= & \left(\frac{1}{\mathcal{M}(v \Psi(N))}-\frac{1}{\mathcal{K}(v \Psi(N))}\right)+ \\
& +(1-N) \frac{v \mathcal{M}^{\prime}(v \Psi(N)) \Psi^{\prime}(N)}{\mathcal{M}^{2}(v \Psi(N))}+\frac{N v \mathcal{K}^{\prime}(v \Psi(N)) \Psi^{\prime}(N)}{c \mathcal{K}^{2}(v \Psi(N))}
\end{aligned}
$$

and we see that, since the first term in the right hand side of (3.10) is positive if $\mathcal{H}(N) \geq 0$ (see (3.8)), the function $\mathcal{H}(N)$ is monotone increasing when positive. Moreover, since

$$
\mathcal{H}(1)=\left(1-\frac{1}{c \mathcal{K}(v \Psi(1))}\right)=1-\frac{1}{\mathcal{R}_{0}(v)},
$$




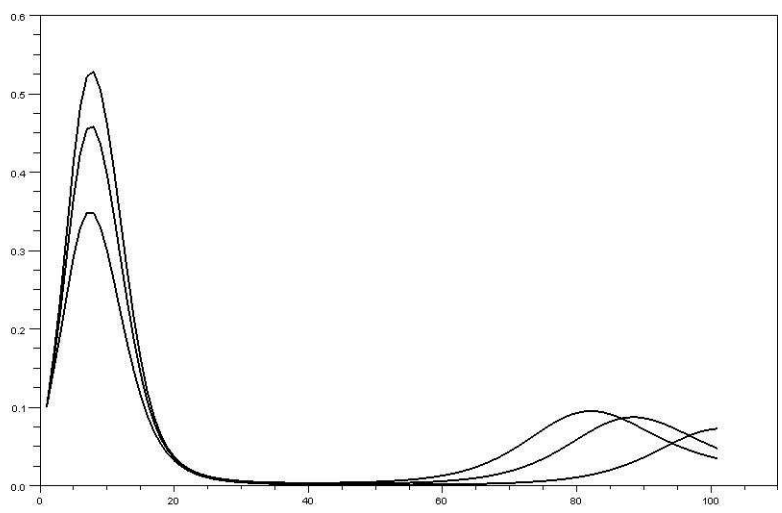

Figure 1. Total infectives $I(t)$ for different values of the (constant) screening strategy $v$. Parameters values are as in (4.3) and (4.4).

we have again that an endemic state exists and is unique, if and only if (3.6) is satisfied.

This parameter $\mathcal{R}_{0}(v)$ is the reproduction number of the infection and gives the threshold condition for the establishment of the infection as endemic. Also, by linearization at the equilibria we can prove the classical result that, if $\mathcal{R}_{0}(v)<1$, only the disease-free equilibrium (3.2) exists and is stable while, if $\mathcal{R}_{0}(v)>1$, the endemic equilibrium (3.7) inherits the stability of the disease-free state which becomes unstable. Of course, $\mathcal{R}_{0}(v)$ is non-increasing with respect to $v$, and

$$
\lim _{v \rightarrow \infty} \mathcal{R}_{0}(v)=c \int_{0}^{\theta_{-}} \omega(\theta) \Gamma(\theta) \Pi(\theta, 0) d \theta
$$

where

$$
\theta_{-}=\inf \{\theta \mid \phi(\theta)>0\} .
$$

Then, a screening strategy at a high constant rate could be effective in eradicating the disease. However, since the strategy cannot go over a certain screening rate because of limited resources, this goal cannot always be attained.

Numerical simulation of system (2.9) with constant values for $v$ may provide epidemic curves showing the effect of a constant screening strategy. The total number of infectives

$$
I(t)=\int_{0}^{\theta_{m}} \Gamma(\theta) u(\theta, t) d \theta
$$

is shown in Figure 1, for different values of the screening variable $v$.

Apart from the effect of constant strategies, the problem of designing time-dependent strategies $v(t)$ must be considered in connection with costs evaluation including both economical and social costs. The remaining Sections are devoted to the definition of such costs and to the discussion of optimal strategies.

\section{Screening strategies}

As we have already discussed in Section 2, in our model (2.7) the screening program performed by health institutions is quantified by the rate $v(t)$, while the success of the program, depending on the functional response of the population, is measured by $v(t) \Psi(N(t)) N(t)$, which gives the actual number of screened 
individuals per unit time. Finally the age distribution of infected individuals found by screening, per unit time, is given by $v(t) \Psi(N(t)) \phi(\theta) i(\theta, t)$. With all these elements we may define the total cost functional to be minimized in order to identify the best strategy compatible with the program purposes. Specifically we consider the following functional:

$$
\begin{aligned}
\Phi(v(\cdot))=\alpha \int_{0}^{T} & \int_{0}^{\theta_{m}} i(\theta, t) d \theta d t+ \\
& \beta \int_{0}^{T} v^{2}(t) d t+\eta \int_{0}^{T} v(t) \Psi(N(t)) N(t) d t+ \\
& \quad+\delta \int_{0}^{T} \int_{0}^{\theta_{m}} c(\theta) v(t) \Psi(N(t)) i(\theta, t) d \theta d t,
\end{aligned}
$$

where the rescaled variables $i(\theta, t)=\Gamma(\theta) u(\theta, t)$ and $N(t)$ depend upon $v(t)$ through the state system (2.9). The different terms in (4.1) account for different costs weighted by the constants $\alpha, \beta, \eta, \delta$. Namely, the first term in (4.1) is a measure of the social cost of the disease: our strategy is, above all, designed

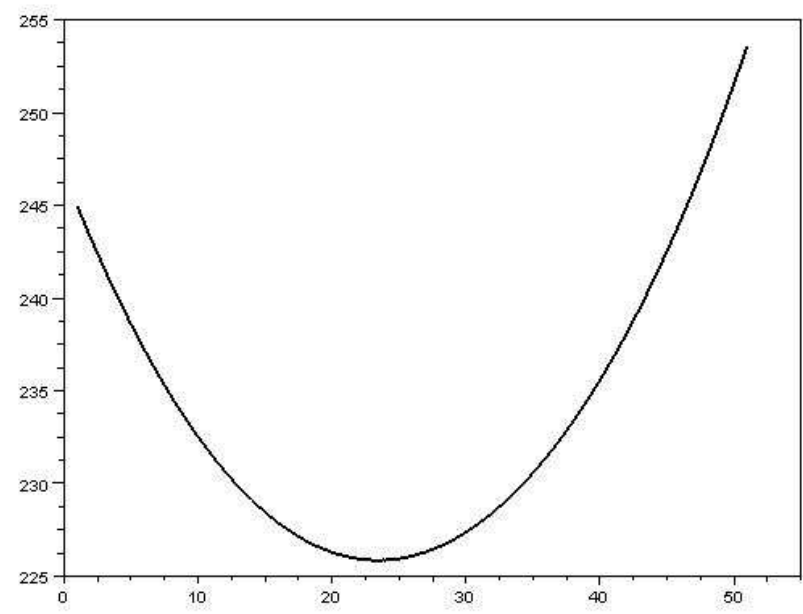

FiguRE 2. The case of a constant strategy $v(t) \equiv v$. The cost function (4.1) is plotted versus the screening strategy $v$ (arbitrary scale). For each value $v$ the cost function $\Phi(v)$ is evaluated after a numerical computation of the state function $i(\theta, t)$ and of the total population $N(t)$, both depending on $v$. Parameters values are shown in (4.3) and (4.4).

to keep the number of infectives as low as possible; then, the second and third terms are a measure of the cost of the screening program, including a fixed cost related to the screening offer and the cost of the actual screening procedure, i.e. the cost of individuals actually tested; finally, the last term in (4.1) gives the cost of the treatments, which is related to the number of infected individuals found by screening. We note that the fixed cost (the second term in (4.1)) is not a linear function of the screening offer $v(t)$ because the effort to reach a large number of people may involve extra expenses that are not necessary when the goal is a low number. Here we have chosen a classical quadratic form borrowed from mechanics, but any increasing function would not imply changes in the mathematical approach presented in Section 5 and in the numerical treatment performed in Section 6.

Our purpose is to minimize the total cost functional over the set of functions $v(t)$ satisfying

$$
0 \leq v(t) \leq v_{m},
$$


where $v_{m}$ is the maximum sustainable effort for screening purposes. As an example, in Figure 2 the cost function (4.1) is calculated in connection with a constant strategy $v(t) \equiv v$ and represented as a function of $v$. The values of the parameters used in the simulations are chosen as

$$
\begin{aligned}
& \text { model parameters : } \quad \theta_{m}=0.1, \quad T=1, \quad v_{m}=1000, \quad c=10^{5} ; \\
& \text { cost function parameters : } \alpha=3150, \quad \beta=0.05, \quad \eta=1, \quad \delta=10 .
\end{aligned}
$$

while, for the functions we used

$$
\begin{aligned}
& \Psi(x)=\frac{1}{1+x}, \quad \Gamma(\theta)=1-\frac{\theta}{\theta_{m}} \\
& \omega(\theta)=10^{-3}(0.8+\theta(10 \theta-1.3)(5.4-100 \theta)) \\
& \nu(\theta) \equiv 0.1, \quad \phi(\theta) \equiv 0.8
\end{aligned}
$$

We may note that, in the case considered in the simulation, the cost is decreasing at low values of $v$, but it is increasing for large ones. Actually, at large values of $v$, the social goal of damping the infection is reached and any further effort only has the effect of rising the cost. In the next Section we consider the problem of existence of an optimal strategy.

\section{Existence of an optimal strategy}

Here we consider the problem of existence of an optimal strategy to the screening problem introduced in the previous sections.

More specifically we look for $v_{*}$ in the convex set

$$
\mathcal{K}=\left\{v \in L^{\infty}(0, T) \mid 0 \leq v(\theta) \leq v_{m}\right\},
$$

and such that

$$
\Phi\left(v_{*}\right)=\min _{v \in \mathcal{K}} \Phi(v) .
$$

As a preliminary step we prove that the solution of the state system (2.9) satisfies some basic estimates, independent of the function $v \in \mathcal{K}$. Towards this goal we consider the total number of infectives (3.12) and note that multiplying the second equation in $(2.9)$ by $\Gamma(\theta)$, and integrating with respect to the variable $\theta$, we get the following equation

$$
\begin{aligned}
I^{\prime}(t)=-\int_{0}^{\theta_{m}} \Gamma(\theta) \frac{\partial u}{\partial \theta}(\theta, t) d \theta-I(t)+ & \\
& -\int_{0}^{\theta_{m}}[\nu(\theta)+v(t) \phi(\theta) \Psi(N(t))] \Gamma(\theta) u(\theta, t) d \theta \\
=\lambda(t) S(t) & -I(t)+ \\
& -\int_{0}^{\theta_{m}}[\gamma(\theta)+\nu(\theta)+v(t) \phi(\theta) \Psi(N(t))] \Gamma(\theta) u(\theta, t) d \theta,
\end{aligned}
$$

where we have used (2.8). Then

$$
I^{\prime}(t) \leq \lambda(t) S(t)-I(t)-\int_{0}^{\theta_{m}} \nu(\theta) \Gamma(\theta) u(\theta, t) d \theta,
$$

so that, using the equations for $S(t)$ and $N(t)$, we get

$$
N^{\prime}(t)-S^{\prime}(t)-I^{\prime}(t) \geq-(N(t)-I(t)-S(t)),
$$


that implies

$$
I(t)+S(t) \leq N(t) \leq 1+(N(0)-1) e^{-t} .
$$

The left hand side inequality in (5.3) follows because

$$
N(0)-S(0)-I(0) \geq 0,
$$

while the right hand side one follows from the third equation in (2.9).

Estimate (5.3) implies that all the components $I(t), S(t), N(t)$ are uniformly bounded with respect to $t \in[0, T]$ and $v \in \mathcal{K}$. Consequently we also have that the functional $\Phi(\cdot)$ is uniformly bounded on all functions $v \in \mathcal{K}$.

The previous estimate (5.3), drives also other important estimates. Namely, using (5.3) in (2.8), we have first

$$
\lambda(t) \leq \frac{c \omega_{+}}{N(t)} I(t) \leq c \omega_{+},
$$

which, used in (2.10) with (5.3), gives also

$$
|u(\theta, t)| \leq \mathcal{M}_{1}=\max \left\{\sup _{\theta \in\left[0, \theta_{m}\right]}\left|u_{0}(\theta)\right|, c \omega_{+}(1+N(0))\right\},
$$

and, from $(2.9, a)$ and $(5.3)$

$$
N(t) \geq S(t) \geq S(0) e^{-\int_{0}^{t}(1+\lambda(s)) d s} \geq S(0) e^{-\left(1+c \omega_{+}\right) t} .
$$

Furthermore, using these estimates in (2.9) and (5.2), we also get

$$
\left|I^{\prime}(t)\right|+\left|S^{\prime}(t)\right|+\left|N^{\prime}(t)\right| \leq \mathcal{M}_{2},
$$

where $\mathcal{M}_{2}$ is a constant independent of $t \in[0, T]$ and $v \in \mathcal{K}$. Finally, since

$$
\begin{aligned}
\lambda^{\prime}(t)=- & \frac{c N^{\prime}(t)}{N^{2}(t)} \int_{0}^{\theta_{m}} \omega(\theta) \Gamma(\theta) u(\theta, t) d \theta+ \\
& +\frac{c}{N(t)} \int_{0}^{\theta_{m}} \omega(\theta) \Gamma(\theta) \frac{\partial u}{\partial t}(\theta, t) d \theta= \\
=- & \frac{N^{\prime}(t)}{N(t)} \lambda(t)-\frac{c}{N(t)} \int_{0}^{\theta_{m}} \omega(\theta) \Gamma(\theta) \frac{\partial u}{\partial \theta}(\theta, t) d \theta-\lambda(t)+ \\
& \quad-\frac{c}{N(t)} \int_{0}^{\theta_{m}}[\nu(\theta)+v(t) \phi(\theta) \Psi(N(t))] \omega(\theta) \Gamma(\theta) u(\theta, t) d \theta= \\
=- & \left(1+\frac{N^{\prime}(t)}{N(t)}-\frac{c \omega(0)}{N(t)} S(t)\right) \lambda(t)+\frac{c}{N(t)} \int_{0}^{\theta_{m}} \omega^{\prime}(\theta) \Gamma(\theta) u(\theta, t) d \theta+ \\
& \quad-\frac{c}{N(t)} \int_{0}^{\theta_{m}}[\gamma(\theta)+\nu(\theta)+v(t) \phi(\theta) \Psi(N(t))] \omega(\theta) \Gamma(\theta) u(\theta, t) d \theta,
\end{aligned}
$$

we use (5.3) - (5.7) to derive

$$
\left|\lambda^{\prime}(t)\right| \leq \mathcal{M}_{3},
$$

where $\mathcal{M}_{3}$ does not depend on $t \in[0, T]$ or $v \in \mathcal{K}$.

The previous estimates allow us to prove existence of an optimal strategy. Let us consider a minimizing sequence $v_{n} \in \mathcal{K}$,

$$
\lim _{n \rightarrow \infty} \Phi\left(v_{n}\right)=\inf _{v \in \mathcal{K}} \Phi(v)
$$

and the respective variables from the state system (2.9), where $v$ is replaced by $v_{n}$,

$$
S_{n}(t), \quad N_{n}(t), \quad u_{n}(\theta, t),
$$




$$
I_{n}(t)=\int_{0}^{\theta_{m}} \Gamma(\theta) u_{n}(\theta, t) d \theta .
$$

The previous variables are also related each other by

$$
u_{n}(\theta, t)=\left\{\begin{array}{ll}
u_{0}(\theta-t) & \text { for } \theta>t \\
\lambda_{n}(t-\theta) S_{n}(t-\theta) & \text { for } \theta \leq t
\end{array}\right\} \times \mathcal{E}\left(\theta, t ; v_{n}, N_{n}\right)
$$

where we have used (2.11) and

$$
\lambda_{n}(t)=\frac{c}{N_{n}(t)} \int_{0}^{\theta_{m}} \omega(\theta) \Gamma(\theta) u_{n}(\theta, t) d \theta
$$

Now, since $v_{n}(t) \leq v_{m}$, the sequence $v_{n}(t)$ is uniformly bounded in $L^{2}(0, T)$ and by estimates (5.7), (5.8) the sequences $S_{n}(t), N_{n}(t), I_{n}(t), \lambda_{n}(t)$ are uniformly Lipschitz continuous, then we can extract a subsequence such that

$$
v_{n} \rightarrow v_{*} \text { weakly in } L^{2}(0, T) .
$$

and

$$
\begin{array}{lll}
S_{n}(t) \rightarrow S_{*}(t) & \text { uniformly in } & {[0, T],} \\
N_{n}(t) \rightarrow N_{*}(t) & \text { uniformly in } & {[0, T],} \\
I_{n}(t) \rightarrow I_{*}(t) & \text { uniformly in } & {[0, T],} \\
\lambda_{n}(t) \rightarrow \lambda_{*}(t) & \text { uniformly in } & {[0, T] .}
\end{array}
$$

We note that, as a consequence we have

$$
\int_{0}^{\theta \wedge t} v_{n}(t-\sigma) \Psi\left(N_{n}(t-\sigma)\right) \phi(\theta-\sigma) d \sigma \rightarrow \int_{0}^{\theta \wedge t} v_{*}(t-\sigma) \Psi\left(N_{*}(t-\sigma)\right) \phi(\theta-\sigma) d \sigma
$$

a.e. in $\left(0, \theta_{m}\right) \times(0, T)$ so that $($ see $(2.11))$

$$
\mathcal{E}\left(\theta, t ; v_{n}, N_{n}\right) \quad \rightarrow \quad \mathcal{E}\left(\theta, t ; v_{*}, N_{*}\right) \quad \text { a.e. in } \quad\left(0, \theta_{m}\right) \times(0, T),
$$

and we can pass to the limit in (5.10) obtaining

$$
u_{n}(\theta, t) \rightarrow u_{*}(\theta, t) \quad \text { a.e. in } \quad\left(0, \theta_{m}\right) \times(0, T),
$$

with

$$
u_{*}(\theta, t)=\left\{\begin{array}{ll}
u_{0}(\theta-t) & \text { for } \quad \theta>t \\
\lambda_{*}(t-\theta) S_{*}(t-\theta) & \text { for } \quad \theta \leq t
\end{array}\right\} \times \mathcal{E}\left(\theta, t ; v_{*}, N_{*}\right) .
$$

Moreover, using $(5.5)$, we can go to the limit in $(5.9),(5.11),(2.9, i),(2.9, \mathrm{c})$

$$
\begin{aligned}
& I_{*}(t)=\int_{0}^{\theta_{m}} \Gamma(\theta) u_{*}(\theta, t) d \theta, \\
& \lambda_{*}(t)=\frac{c}{N_{*}(t)} \int_{0}^{\theta_{m}} \omega(\theta) \Gamma(\theta) u_{*}(\theta, t) d \theta, \\
& S_{*}^{\prime}=\Lambda-\lambda_{*}(t) S_{*}(t)-\mu S_{*}(t), \\
& N_{*}^{\prime}=\Lambda-\mu N_{*}(t)-\int_{0}^{\theta_{n}} \nu(\theta) \Gamma(\theta) u_{*}(\theta, t) d \theta,
\end{aligned}
$$

so that $S_{*}, N_{*}, u_{*}$ is the solution to (2.9) corresponding to $v_{*}$. Finally from (4.1) we have

$$
\lim _{n \rightarrow \infty} \Phi\left(v_{n}\right)=\Phi\left(v_{*}\right)
$$

so that $v_{*}$ satisfies (5.1). 


\section{The optimality system}

For the optimal screening strategy (5.1), we introduce the formal Lagrange function

$$
\begin{gathered}
\mathcal{L}(v, S, u, N, p, q, r, z)=\Phi(v)+\int_{0}^{T}\left(S^{\prime}(t)-1-\lambda(t) S(t)-S(t)\right) p(t) d t \\
+\int_{0}^{T} \int_{0}^{\theta_{m}}\left(\frac{\partial u}{\partial t}+\frac{\partial u}{\partial \theta}+[1+\nu(\theta)+v(t) \Psi(N(t)) \phi(\theta)] u(\theta, t)\right) q(t, \theta) d t d \theta \\
+\int_{0}^{T}\left(N^{\prime}(t)-1+N(t)+\int_{0}^{\theta_{m}} \nu(\theta) u(\theta, t) d \theta\right) r(t) d t+\int_{0}^{T}(i(0, t)-\lambda(t) S(t)) z(t) d t
\end{gathered}
$$

with multipliers $p \in L^{2}((0, T)), q \in L^{2}\left((0, T) \times\left(0, \theta_{m}\right), r \in L^{2}((0, T))\right.$ and $z \in L^{2}((0, T))$. Let us now consider the variables $v, S, u, N, p, q, r, z$ as independent and compute and set equal to zero the derivatives of $\mathcal{L}$, with respect to the seven last variables. After some computations we obtain:

$$
\left\{\begin{aligned}
a) & -p^{\prime}(t)=\lambda(t)(p+q(0, t))+p(t) \\
b) & -\frac{\partial q}{\partial t}-\frac{\partial q}{\partial \theta}+[1+\nu(\theta)+v(t) \Psi(N(t)) \phi(\theta)] q(\theta, t)= \\
& -\alpha-\delta c(\theta) v(t) \Psi(N)-\nu(\theta) r(t) \\
& +\frac{c(\theta)}{N(t)} \omega(\theta) S(t)(p(t)+q(0, t)) \\
c) & r^{\prime}(t)=r(t)+\frac{\lambda(t)}{N(t)} S(t)(p(t)+q(0, t)) \\
& +\eta v(t)\left(\Psi^{\prime}(N) N+\Psi(N)\right) \\
& +\left(\delta \int_{0}^{\theta_{m}} c(\theta) u(\theta, t) d \theta+\int_{0}^{\theta_{m}} \phi(\theta) u(\theta, t) q(\theta, t) d \theta\right) \Psi^{\prime}(N) v(t),
\end{aligned}\right.
$$

complemented by the transversality conditions

$$
p(T)=0, \quad q(\theta, T)=0, \quad q\left(\theta_{m}, t\right)=0, \quad r(T)=0
$$

in addition to the state equations (2.7). System (6.1) supplemented by the transversality conditions is called the adjoint system of the optimal control problem.

When computing the derivative of the Lagrangian with respect to the first variable, we obtain the gradient of $\Phi$.

$$
\begin{aligned}
& \nabla_{v}(\Phi)=2 \beta v+\eta \Psi(N) N \\
& +\left(\delta \int_{0}^{\theta_{m}} c(\theta) u(\theta, t) d \theta+\int_{0}^{\theta_{m}} \phi(\theta) u(\theta, t) q(\theta, t) d \theta\right) \Psi(N)
\end{aligned}
$$

Based on (6.1) and (6.2) we can approximate the optimal screening strategy using a discretization of the following algorithm:

- Step 0 Choose a control $v(t)$ randomly.

- Step 1 Compute the solution of the state equations (2.7).

- Step 2 Compute the solution of the adjoint equation (6.1).

- Step 3 Compute the gradient of $\Phi$, by (6.2).

- Step 4 Compute the new control using a nonlinear gradient method.

- Step 5 Project the control on the admissible domain (constraints).

- Step 6 If the norm of the gradient of $\Phi$ is less than some tolerance then stop; else return to step 1. 


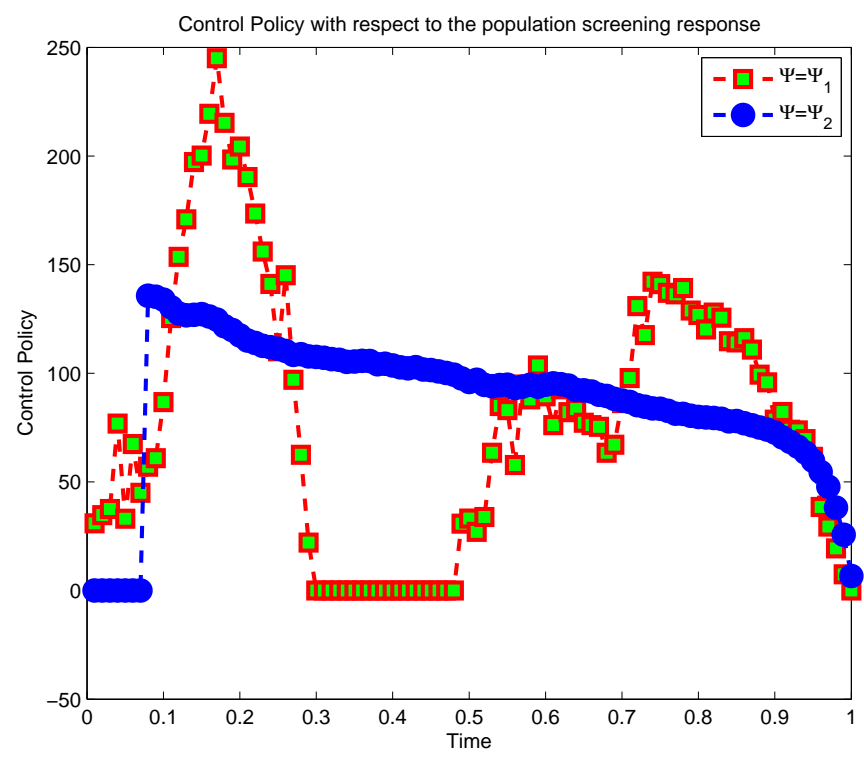

Figure 3. The optimal strategy $v(t)$ for different forms of the functional response $\Psi(N)$. Other parameters and functions are as in (6.3) and (6.4).

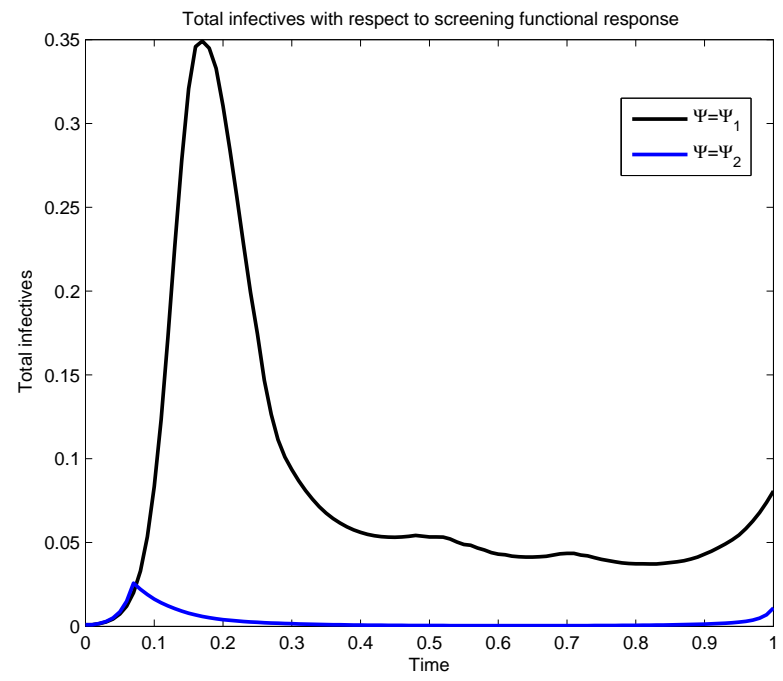

Figure 4. Total infectives relative to the different screening strategies shown in Figure 3

We have actually used this procedure to run some numerical simulations and illustrate the effect of agedisease structure on the optimal screening strategy. More specifically, in discretizing the algorithm just described, we used a semi-implicit scheme on the characteristic lines to solve the state equations; the nonlinear renewal equation for the infected population was solved explicitly. Besides, the cost function was discretized using the trapezoidal rule and a discrete Lagrange function was also obtained by using 
the trapezoidal rule and the discretized state equation. From this discretized Lagrangian we derive, in the discrete context, a discrete adjoint equation that we use in the computations (this means that we do not obtain the discrete adjoint equation directly from the continuous one). The numerical parameters used to produce Figures 3 and 4 are:

$$
\begin{aligned}
& \text { model parameters : } \quad \theta_{m}=0.1, \quad T=1, \quad v_{m}=1000, \quad c=10^{5} ; \\
& \text { cost function parameters : } \alpha=3150, \quad \beta=0.005, \quad \eta=0.1, \quad \delta=10 .
\end{aligned}
$$

while, for the functions we used

$$
\begin{aligned}
& \Psi_{1}(N)=\frac{1}{1+10 N}, \Psi_{2}(N)=\frac{1}{1+0.1 N} \quad \Gamma(\theta)=1-\frac{\theta}{\theta_{m}} \\
& \omega(\theta)=10^{-3}(0.8+\theta(10 \theta-1.3)(5.4-100 \theta)) \\
& \nu(\theta) \equiv 0.1, \quad \phi(\theta) \equiv 0.8
\end{aligned}
$$

In Figures 3, 4 we see the effect of the population screening response on the optimal control strategy. We see (Figure 3 ) that for $\Psi_{1}(N)$ the optimal strategy prescribes no screening in the middle of the campaign , while for $\Psi_{2}(N)$ there is no screening at the beginning. Moreover, the optimal screening is never at the maximum allowable value $v_{m}$. In Figure 4 we see that for $\Psi_{1}(N)$ the epidemics persists while for $\Psi_{2}(N)$ it disappears. In Figure 5, we examine the effect of the disease detectability, choosing different

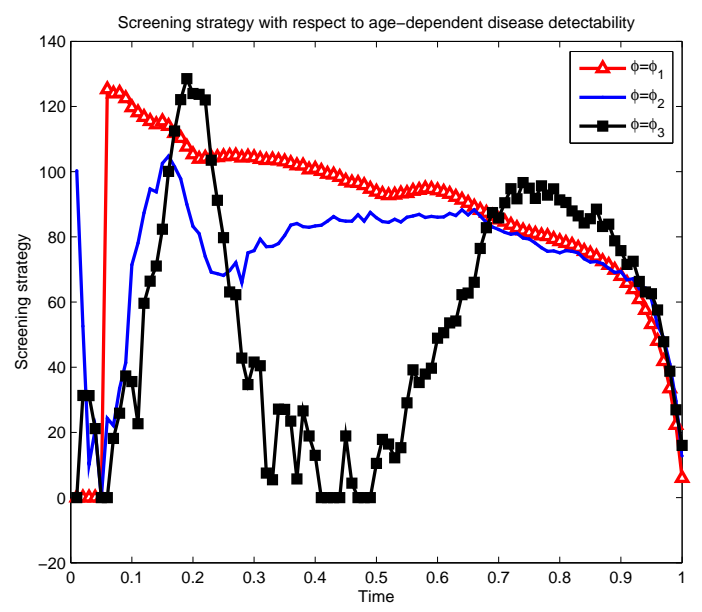

FiguRE 5 . The optimal strategy $v(t)$ for different forms of the function $\phi(\theta)$

age-dependent functions $\phi(\theta)$, with equal mean, and keeping the other choices as in (6.3), (4.4). Namely, we consider the following cases

$$
\phi_{1}(\theta) \equiv 0.08, \quad \phi_{2}(\theta)=\chi_{(0.02,0.1)}(\theta), \quad \phi_{3}(\theta)=\frac{4}{3} \chi_{(0.04,0.1)}(\theta),
$$

and we see that with $\phi_{1}(\theta)$, when the disease detectability is possible for all ages, the optimal screening strategy has no screening at the beginning. Instead, when detectability is not possible for small ages, the screening effort should mainly concern the beginning and the end of the campaign. 
Finally, in Figure 6, we examine the optimal screening strategy with respect to different disease-age infectiveness functions, $\omega(\theta)$, with equal mean value, while the remaining parameters are still chosen as in (6.3), (4.4). Thus, in addition to the function $\omega_{1}(\theta)=\omega(\theta)$ defined in (4.4), we consider the following options

$$
\omega_{2}(\theta) \equiv 810^{-4}, \quad \omega_{3}(\theta)=0.04 \chi_{(0.02,0.1)}(\theta), \quad \omega_{4}(\theta)=0.02 \chi_{(0.04,0.1)}(\theta)
$$

For $\omega_{1}(\theta)$ and for the constant function $\omega_{2}(\theta)$ we have a very similar optimal screening strategy, starting from zero at the beginning of the program, increasing to a maximum screening effort, and then decreasing to zero at the end of the program. For the two other examples, $\omega_{3}(\theta)$ and $\omega_{4}(\theta)$ we have some very similar optimal screening strategies, both suggesting a strong effort at the beginning of the campaign.

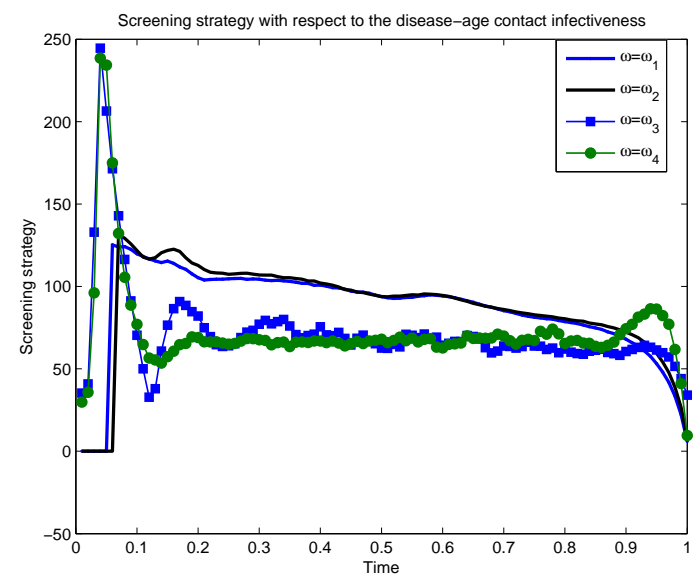

Figure 6 . The optimal strategy $v(t)$ for different forms of the infectiveness $\omega(\theta)$

\section{Conclusions}

We have presented a model for investigating optimal screening strategies within the context of diseases characterized by appreciable duration of the infectiveness period and by variability of the transmission risk. The analysis of the model showed that disease endemicity is possible even when a high screening effort is exerted. Then a cost function (including both social cost and economical spending) was introduced in order to find an optimal screening campaign minimizing the cost. We proved that an optimal strategy does exists and we set up a numerical method to explore different situations due to different shapes and parameter ranges in the structure of the model. In fact, Figures 3-6 show that the population response to the screening offer (namely function $\Psi(N)$ ), the disease detectability together with cure and/or prevention efficacy (namely function $\phi(\theta)$ ) and the disease progression and infectiveness structure (namely function $\omega(\theta)$ ), strongly infuence the optimal screening campaign. The numerical simulations may produce different strategies, in some cases suggesting a non-uniform intervention and, in all cases the effort does not reach the maximum a priori designed as an upper bound. A general conclusion that may be drawn from this study is that a model based on class-age may be better fitted to approach diseases with long incubation and/or latency periods. In fact, in the simulations we see that the differences in the optimal strategies are due to the different dependence of the functions upon class-age. Functions and parameters introduced in the model are only based on theoretical assumptions, but they could be designed in correspondence with specific diseases. The case of the HIV-AIDS infection could be realistically 
approached if sociological data and clinical information could be quantified to give the model a realistic shape.

\section{References}

[1] C. Almeder, G. Feichtinger, W. C. Sanderson, V. M. Veliov. Prevention and medication of HIV/AIDS: the case of Botswana, Central European J. Oper. Res. 15 (2007), 47-61.

[2] S. Anita. Analysis and Control of Age-Dependent Population Dynamics, Kluwer, Boston, MA, 2000.

[3] S. Anita, M. Iannelli, M.Y. Kim, E.J. Park. Optimal harvesting for periodic age-dependent population dynamics, SIAM J. Appl. Math. 58 (1999), 1648-1666.

[4] B. Armbruster, M. L. Brandeau. Optimal mix of screening and contact tracing for endemic diseases, Math. Biosci. 209 (2007) 386-402.

[5] F. Ball, N. G. Becker. Control of transmission with two types of infection, Math. Biosci. 200 (2006) $170-187$.

[6] V. Barbu, M. Iannelli. Controlling the SIS Epidemics, Proceedings of the Conference on Mathematical Models in Medical and Health Sciences, Nashville, Tennessee, edited by M. A. Horn, G. Simonett, G. F. Webb, 1998.

[7] V. Barbu, M. Iannelli. Optimal control of population dynamics, J. Optim. Theory Appl. 102 (1999), 1-14.

[8] H. Behncke. Optimal control of deterministic epidemics, Optim. Control Appl. Meth. 21 (2000), 269-285.

[9] K. W. Blayneh, A. B. Gumel, S. Lenhart, T. Clayton. Backward Bifurcation and Optimal Control in Transmission Dynamics of West Nile Virus, Bulletin of Mathematical Biology, 72 (2010), 1006-1028.

[10] C. Castillo-Chavez, Z. Feng. Global stability of an age structured model for TB and its applications to optimal vaccination strategies, Math. Biosci. 151 (1998), 135-154.

[11] G. Feichtinger, V. M. Veliov, T. Tsachev. Maximum principle for age and duration structured systems: a tool for optimal prevention and treatment of HIV, Mathematical Population Studies, 11 (2004), 3-28.

[12] Z. Feng, H. R. Thieme. Recurrent outbreaks of childhood diseases revisited: the impact of isolation, Math. Biosci. 128 (1995), 93-130.

[13] K. R. Fister, S. Lenhart. Optimal control of a competitive system with age-structure, J. Math. Anal. Appl. 291 (2004), $526-537$.

[14] K.P. Hadeler, J. Muller. Optimal harvesting and optimal vaccination, Math. Biosci. 206 (2007), $249-272$.

[15] E. Hansen, T. Day. Optimal control of epidemics with limited resources, J. Math. Biol. 62 (2011), $423-451$.

[16] H. W. Hethcote, J. A. York. Gonorrhea Transmission and Control, Lectures Notes in Biomathematics 56, Springer Verlag 1984.

[17] H. W. Hethcote. Optimal ages of vaccination for measles, Math. Biosci. 89 (1988), 29-52.

[18] H. W. Hethcote, P. Waltman. Optimal vaccination schedules in a deterministic epidemic model, Math. Biosci. 18 (1973), 365-381.

[19] N. Hritonenko, Y. Yatsenko. The structure of optimal time- and age-dependent harvesting in the Lotka McKendrik population model, Math. Biosci. 208 (2007), 48-62.

[20] J. M. Hyman, J. Li, E. A. Stanley. Modeling the impact of random screening and contact tracing in reducing the spread of HIV, Math. Biosci. 181 (2003), 17-54.

[21] E. Jung, S. Lenhart, Z. Feng. Optimal Control of Treatments in a Two Strain Tuberculosis Model, Discrete and Continuous Dynamical Systems 2 (2002), 473-482.

[22] S. M. Kassa, A. Ouhinou. Epidemiological models with prevalence dependent endogenous self-protection measure, Math. Biosci. 229 (2011), 41-49.

[23] X. Liu, P. Stechlinski. Pulse and constant control schemes for epidemic models with seasonality, Nonlinear Analysis: RWA 12 (2011), 931-946.

[24] F.A. Milner and R. Zhao. A new mathematical model of syphilis, Math. Model. Nat. Phenom. 5 (2010), 96-108.

[25] R. Morton, K. H. Wickwire On the optimal control of a deterministic epidemic, Adv. Appl. Prob. 6 (1974), $622-635$.

[26] A. Mubayi, C. Kribs Zaleta, M. Martcheva, C. Castillo-Chavez. A cost based comparison of quarantine strategies for new emerging diseases, Math. Biosc. Eng. 7 (2010), 687-717.

[27] J. Muller, M. Kretzschmar, K. Dietz. Contact tracing in stochastic and deterministic epidemic models, Math. Biosci. 164 (2000), 39-64.

[28] M. L. Ndeffo Mbah, C. A. Gilligan. Optimization of control strategies for epidemics in heterogeneous populations with symmetric and asymmetric transmission, J. Theor. Biology 262 (2010), 757-763.

[29] J. L. Sanders. Quantitative guidelines for communicable disease control programs, Biometrics, 27 (1971), $883-893$.

[30] A. J. Terry. Pulse Vaccination Strategies in a Metapopulation SIR model, Math. Biosc. Eng. 7 (2010), 455-477.

[31] K. H. Wickwire. A note on the optimal control of carrier-borne epidemics, J. Appl. Prob. 12 (1975), 565-568.

[32] K. H. Wickwire. Optimal isolation policies for deterministic and stochastic epidemics, Math. Biosci. 26 (1975), $325-346$.

[33] K. H. Wickwire. Mathematical models for the control of pests and infectious diseases: a survey, Theor. Pop. Biol. 11 (1977), 182-238. 